\title{
Dystrophic heart failure blocked by membrane sealant poloxamer
}

\author{
Soichiro Yasuda ${ }^{1 \star}$, DeWayne Townsend $^{1 \star}$, Daniel E. Michele ${ }^{1,2}$, Elizabeth G. Favre ${ }^{1}$, Sharlene M. Day ${ }^{2}$ \\ \& Joseph M. Metzger ${ }^{1,2}$
}

Dystrophin deficiency causes Duchenne muscular dystrophy (DMD) in humans, an inherited and progressive disease of striated muscle deterioration that frequently involves pronounced cardiomyopathy ${ }^{1}$. Heart failure is the second leading cause of fatalities in $\mathrm{DMD}^{1,2}$. Progress towards defining the molecular basis of disease in DMD has mostly come from studies on skeletal muscle, with comparatively little attention directed to cardiac muscle. The pathophysiological mechanisms involved in cardiac myocytes may differ significantly from skeletal myofibres; this is underscored by the presence of significant cardiac disease in patients with truncated or reduced levels of dystrophin but without skeletal muscle disease ${ }^{3}$. Here we show that intact, isolated dystrophin-deficient cardiac myocytes have reduced compliance and increased susceptibility to stretch-mediated calcium overload, leading to cell contracture and death, and that application of the membrane sealant poloxamer 188 corrects these defects in vitro. In vivo administration of poloxamer 188 to dystrophic mice instantly improved ventricular geometry and blocked the development of acute cardiac failure during a dobutaminemediated stress protocol. Once issues relating to optimal dosing and long-term effects of poloxamer 188 in humans have been resolved, chemical-based membrane sealants could represent a new therapeutic approach for preventing or reversing the progression of cardiomyopathy and heart failure in muscular dystrophy.

It is unknown whether dystrophin deficiency directly causes altered force transmission and/or membrane fragility in cardiac muscle at the single myocyte level. Here we used a unique microcarbon fibre technique (Supplementary Fig. 1 and Video 1) that enables the simultaneous assessment of force and intracellular calcium concentrations of isolated, membrane-intact myocytes under physiologically relevant mechanical loading. Single, membrane-intact adult cardiac myocytes from dystrophin-deficient mice $\left(m d x\right.$, also known as $\left.D m d^{m d x}\right)$ and control mice were passively stretched (1-s duration) over a physiologically relevant range of sarcomere lengths (1.75-2.20 $\mu \mathrm{m}$; ref. 4$)$, and passive tension and intracellular calcium concentrations $\left(\left[\mathrm{Ca}^{2+}\right]_{\mathrm{i}}\right)$ were recorded. Figure 1a shows photomicrographs of control and $m d x$ myocytes at different stages of the stretch protocol. In Fig. 1b, representative simultaneous recordings of length, tension and Fura-2 ratio of single cardiac myocytes from a control and $m d x$ mouse are shown. The traces on the left are the active twitch tension and calcium transient during an electrically stimulated isometric contraction at a resting sarcomere length of $1.75-1.80 \mu \mathrm{m}$. Remaining traces are tension and calcium recordings during passive stretching of the myocyte from a resting sarcomere length of $1.75 \mu \mathrm{m}(0 \%$ stretch $)$ to a physiologically relevant diastolic sarcomere length of $2.00-2.20 \mu \mathrm{m}$ (20\% stretch). Peak isometric twitch tension is not different between control and $m d x$ myocytes $\left(3.6 \pm 0.7\right.$ and $4.4 \pm 0.8 \mathrm{mN} \mathrm{mm}^{-2}$, respectively; mean \pm s.e.m; $P=0.47)$, indicating that excitation-contraction coupling and force generation/transmission are normal in $m d x$ myocytes. However, dystrophin deficiency did have significant effects on passive physiological stretches in myocyte sarcomere length (Fig. 1b). With passive excursions from resting sarcomere lengths of $1.80 \mu \mathrm{m}$ to longer sarcomere lengths, $m d x$ myocytes developed significantly increased tension compared with control myocytes (Fig. 2a). This is in contrast to previous studies on skeletal myotubes, where dystrophic muscles were found to be more compliant than control muscles ${ }^{5}$. Stretches to sarcomere lengths greater than $2.10 \mu \mathrm{m}$ resulted in $m d x$ myocytes becoming unstable, with increased $\left[\mathrm{Ca}^{2+}\right]_{\mathrm{i}}$, fibrillations, eventual calcium overload and subsequent contracture and myocyte death (Fig. 1 and Supplementary Video 2). Reducing extracellular $\left[\mathrm{Ca}^{2+}\right.$ ] from 1.8 to $0.2 \mathrm{mM}$ shifted the tension-extension curves rightward, permitting stretches of up to $2.30 \mu \mathrm{m}$ in both $m d x$ and control myocytes (data not shown). This highlights a role for extracellular calcium in mediating the reduced compliance of $m d x$ myocytes.

These results establish a primary defect in cell compliance in single, isolated $m d x$ myocytes, which show increased susceptibility to stretch-mediated membrane instability and calcium-dependent hyper-contracture. We next tested whether a chemical-based membrane repair approach would have efficacy in isolated $m d x$ myocytes. The nonionic triblock co-polymer poloxamer 188, poly(ethylene oxide) ${ }_{80}$-poly(propylene oxide) ${ }_{27}$-poly(ethylene oxide) ${ }_{80}$ (molecular mass $\sim 8.4 \mathrm{kDa}$ ), which has previously been shown to insert into artificial lipid monolayers and repair damaged biological membranes $^{6,7}$, was assessed for its ability to stabilize $m d x$ myocyte membranes during physiological loading conditions in vitro (Fig. 2a). Within the physiologically relevant sarcomere length range of $1.80-2.20 \mu \mathrm{m}, 150 \mu \mathrm{M}$ P188 (ref. 8) fully restored $m d x$ myocyte compliance and $\left[\mathrm{Ca}^{2+}\right]_{\mathrm{i}}$ to control levels (Fig. 2). This dose of P188 was shown to be effective in protecting skeletal muscle from electrocution damage ${ }^{8}$. P188 also significantly improved twitch tension performance after a lengthening contraction (Supplementary Fig. 2). P188 had no effect on control C57BL/10 myocyte compliance within this sarcomere length range $(P=0.49$ at $2.0 \mu \mathrm{m}$ and $P=0.58$ at $2.1 \mu \mathrm{m}$; Fig. 2a).

The increase in $\left[\mathrm{Ca}^{2+}\right]_{\mathrm{i}}$ resulting from passive stretch is significantly greater in $m d x$ myocytes, and this is corrected by P188 (Fig. 2b). To further address the mechanism of calcium entry, myocytes were treated with the L-type $\mathrm{Ca}^{2+}$ channel blocker nifedipine. In contrast to $\mathrm{P} 188$, heightened $\left[\mathrm{Ca}^{2+}\right]_{i}$ upon stretch was not blocked by nifedipine (Fig. 2b), suggesting that elevated $\left[\mathrm{Ca}^{2+}\right]_{\mathrm{i}}$ may arise from channels that are not sensitive to dihydropyridine (DHP). Calcium leak channels are another possible pathway of calcium entry, but are not activated by stretch ${ }^{9}$. The most likely mechanism of 
calcium entry is through small tears in the membrane that are prevented by $\mathrm{P} 188$ in $m d x$ myocytes. In additional experiments, a lower concentration of P188 $(60 \mu \mathrm{M})$, previously shown to enhance membrane resealing in toxin-poisoned fibroblasts ${ }^{10}$, significantly improved $m d x$ myocyte stability during passive stretch (Fig. 2c).

To test directly whether myocyte stretching causes membrane damage in $m d x$ myocytes, the styryl dye FM 1-43 was used. This compound is impermeable to intact cell membranes, and specifically fluoresces when incorporated into the lipidic environment of

a

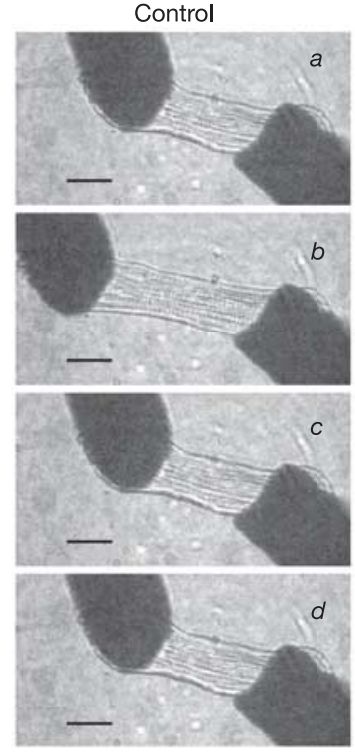

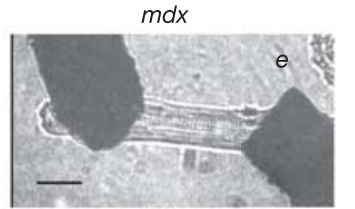
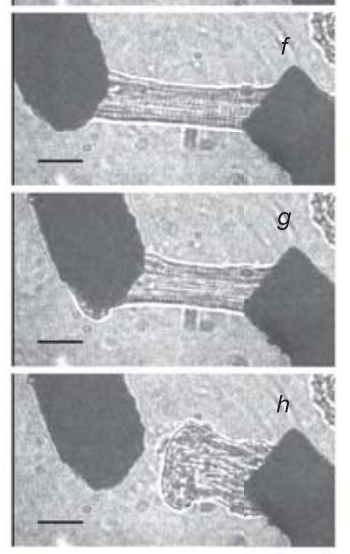

b

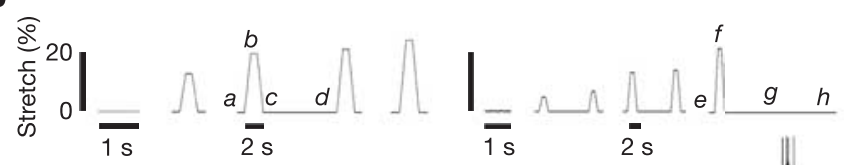

cellular membranes. FM 1-43 has been used to demonstrate membrane disruption/repair in a range of cell systems ${ }^{10}$, including dysferlin-deficient skeletal muscle fibres ${ }^{11}$. We subjected single cardiac myocytes to a single stretch of $20 \%$ in the presence of $2.5 \mu \mathrm{M}$ FM 1-43 (Fig. 2d). Control C57BL/10 myocytes were mechanically stable after stretch, with no change in FM 1-43 fluorescence. In contrast, $m d x$ myocytes became unstable after stretch, showing fibrillations (Supplementary Video 3) but not cell contracture (Fig. 1a, label $h$ ), and showing a concurrent steady increase in FM 1-43 fluorescence. Stretch-mediated $m d x$ myocyte instability/ fibrillation and elevated FM 1-43 fluorescence were completely blocked by P188. We hypothesize that a $20 \%$ stretch causes instability of the $m d x$ myocyte, allowing transmembrane influx of $\mathrm{Ca}^{2+}$ that triggers cycles of myocyte fibrillations, which in turn create more membrane instability. We interpret the steady increase in FM 1-43 fluorescence after the triggering stretch as the accumulation of dye incorporated into the plasma membrane owing to continuous sarcolemmal disruptions of the fibrillating myocyte. The time course of FM 1-43 fluorescence in $m d x$ myocytes after stretch differs somewhat from the biphasic increase in fluorescence observed in skeletal muscles after a single laser-generated pore in the membrane ${ }^{11}$. The type of inciting damage (laser focal point damage in skeletal muscle versus stretch of entire cardiac myocyte in this study) and differences in calcium handling between cardiac and skeletal muscles (calcium-induced calcium release being significantly more prominent in cardiac than skeletal muscle) probably account for the differing kinetics of FM 1-43 observed here. We also found that stretching $m d x$ myocytes by more than $20 \%$ could cause fibrillations and elevated FM 1-43 fluorescence even in the presence of P188, indicating that the fluorescence properties of FM 1-43 are not altered by P188. From these results we conclude that P188 enhances sealing of the $m d x$ plasma membrane sufficiently to prevent the initial stretch-induced injury from causing cycles of membrane de-stabilization, $\mathrm{Ca}^{2+}$ entry and fibrillation.

We next determined whether the cellular effects of P188 in preventing stretch-induced membrane damage in $m d x$ cardiac myocytes would translate to preventing cardiac dysfunction in $m d x$ mice in vivo. Baseline left ventricular haemodynamic performance was depressed in $m d x$ mice, including reduced left ventricular enddiastolic volume (LVEDV) (Fig. 3 and Supplementary Table 1). Pre-treatment with P188 by intravenous infusion increased LVEDV to levels seen in control hearts. We hypothesize that the lower LVEDV in $m d x$ hearts is an organ-level manifestation of the membrane defect observed in single isolated myocytes, and that the acute rescue of LVEDV by $\mathrm{P} 188$ in the $m d x$ heart is a direct effect of restoring normal compliance in single $m d x$ cardiac myocytes (Fig. 2). The haemodynamic parameters (Supplementary Table 1) indicate that the primary effect of acute P188 administration is to restore enddiastolic volumes without significantly changing end-diastolic pressure or other parameters, consistent with a role for P188 in improving the compliance of the $m d x$ cardiac myocytes in vitro (Fig. 2).

Acute cardiomyopathy and heart failure can be induced by cardiovascular stressors in $m d x$ mice $^{12}$. We therefore tested whether an acute dobutamine stress challenge in vivo could cause acute cardiac failure, and whether this phenotype could be blocked by P188. Untreated $m d x$ mice had a very attenuated response to dobutamine infusion (data not shown) and a significant incidence of acute cardiac failure (Fig. 3c) during the 30-min stress-test regime, whereas control mice responded robustly to dobutamine infusion and did not develop acute heart failure. Pre-treatment of $m d x$ mice by intravenous infusion of P188 immediately improved the haemodynamic response to dobutamine infusion (data not shown) and conferred protection from dobutamine-induced acute heart failure in vivo $(P=0.005$, Fig. $3 c)$.

Collectively, these findings demonstrate that in dystrophinhyper-contracture and death $(h)$ 
deficient hearts, abnormal stretch-induced increases in $\left[\mathrm{Ca}^{2+}\right]_{\mathrm{i}}$ result in decreased compliance at the cellular level and lower diastolic volume in vivo. The ability of the membrane sealant $\mathrm{P} 188$ to correct these abnormalities suggests that the calcium influx results from a loss of membrane integrity. Endogenous mechanisms of membrane repair have recently been investigated in dysferlin-deficient dystrophic skeletal muscles ${ }^{11}$, and some of these repair mechanisms are elevated as part of the compensatory response in skeletal muscles of $m d x$ mice $^{13}$. It is reasonable to assume that similar mechanisms are present within the cardiomyocytes, but that these are not sufficient to redress membrane instability, particularly under heightened mechanical stress.

Current therapeutic strategies for Duchenne muscular dystrophy are focused on the expression of dystrophin (through exon skipping or viral transduction of truncated dystrophin) or other genes (for
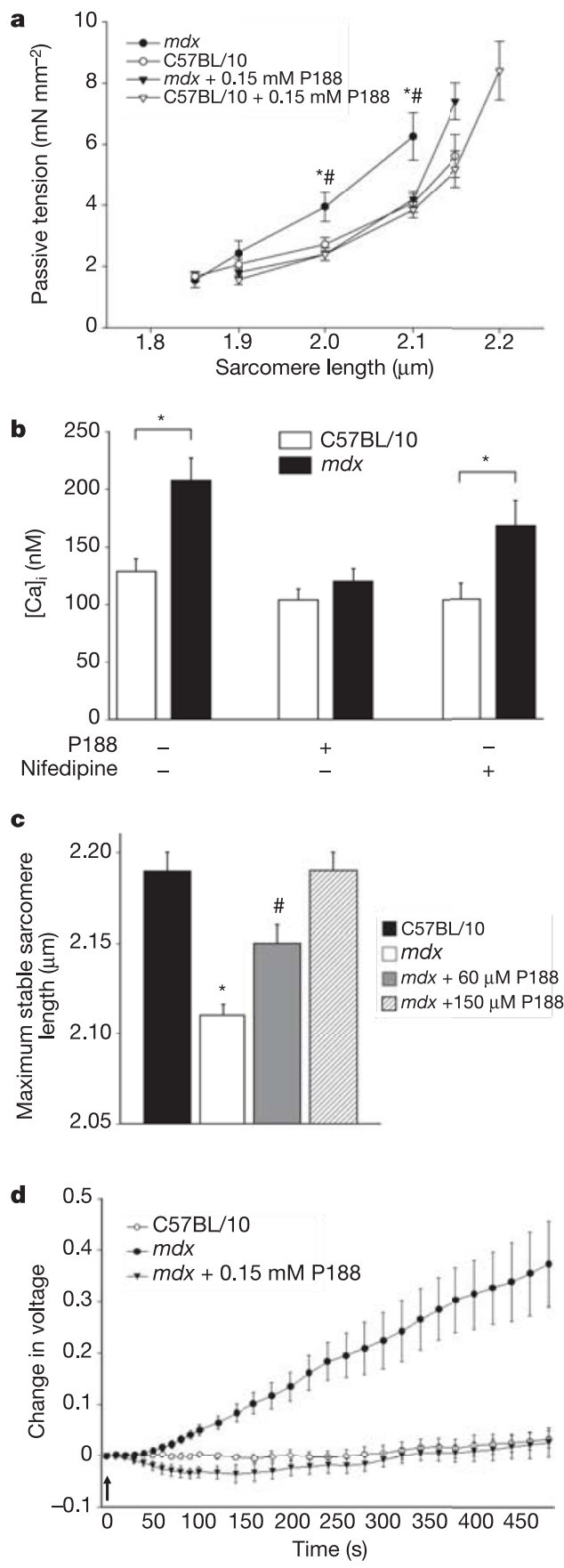

example, utrophin or dysferlin) that limit the consequences of dystrophin deficiency ${ }^{14-17}$. The present results suggest a comparatively simple, chemical-based alternative involving membrane stabilization and/or repair by intravenous administration of P188. As shown here in this animal model of DMD, P188 can have immediate beneficial haemodynamic effects under basal conditions, and may prevent myocardial damage under acute stress conditions, for example during surgery in DMD patients. Currently, P188 is in phase III clinical trials for the treatment of vaso-occlusive crises in sickle-cell anaemia patients, having recently demonstrated the safety and non-toxicity of P188 for short-term (24-h) use in humans ${ }^{18}$. Unlike the episodic course of sickle-cell anaemia, DMD is a progressive disease, and effective P188 therapy would probably require chronic intravascular administration. Long-term effects of this sealant are unclear at present and would have to be validated in large-animal models of muscular dystrophy. In one safety study designed for $72 \mathrm{~h}$ the highest dose tested was terminated early because of adverse affects (primarily muscle pain) in healthy volunteers, indicating that high doses may be too toxic for long-term use ${ }^{19}$. Human studies will be required to fully address the optimal dose, route and duration (acute and chronic) for delivery of chemical membrane sealants. If issues of dosing and long-term safety can be addressed, our results indicate that membrane-sealing poloxamers could represent a new class of therapeutic agents for preventing or limiting progressive damage to the hearts of DMD patients, and might also have benefits in other cardiomyopathies associated with defects in the dystrophin glycoprotein complex ${ }^{20}$.

\section{METHODS}

Animals. Control (C57BL/10 SnJ) and $m d x(\mathrm{C} 57 \mathrm{BL} / 10 \mathrm{ScSn}-m d x)$ mice obtained from Jackson Laboratories were maintained in barrier isolation facilities at the University of Michigan. The procedures used in this study were approved by the University of Michigan Committee on the Use and Care of Animals.

Measurements of cardiac myocyte length, tension and $\left[\mathrm{Ca}^{2+}\right]_{\mathrm{i}}$. Methods for isolating mouse adult cardiac myocytes were as previously reported ${ }^{21}$. Isolated single myocytes were transferred to an experimental chamber containing a silicon-coated glass bottom and platinum electrodes mounted on the sides to electrically stimulate the myocytes. The chamber was mounted on the stage of an inverted microscope (Nikon TE300, $\times 40$ objective) and kept at $37^{\circ} \mathrm{C}$ with a thermoelectric device. The system for measuring length, tension and $\left[\mathrm{Ca}^{2+}\right]_{\mathrm{i}}$ in membrane-intact cardiac myocytes was developed from a previously reported method $^{22}$, using a pair of microcarbon fibres. One fibre was connected to a sensitive force transducer system $\left(200 \mathrm{mV} \mathrm{mg}^{-1}\right.$, Aurora Scientific) and the other was connected to a piezoelectric translator (P-173, PI Polytec) to control

Figure 2 | Effects of $\mathrm{P} 188$ on passive tension and $\left[\mathrm{Ca}^{2+}\right]_{i}$ during sarcomere length stretch in control and $\boldsymbol{m d x}$ single cardiac myocytes. a, Effects of P188 on passive tension-extension relationships. Mdx myocytes have significantly higher passive tension than control $(\mathrm{C} 57 \mathrm{BL} / 10)$ myocytes (asterisk, $P=0.038$ and $P=0.031$ at sarcomere lengths 2.0 and $2.1 \mu \mathrm{m}$, respectively). Treatment with P188 significantly reduces tension compared with untreated $m d x$ myocytes (hash symbol, $P=0.021$ and $P=0.040$ at sarcomere lengths 2.0 and $2.1 \mu \mathrm{m}$, respectively). b, Summary of the effects of $\mathrm{P} 188$ and nifedipine on $\left[\mathrm{Ca}^{2+}\right]_{\mathrm{i}}$ in myocytes upon sarcomere length stretch to $2.1 \mu \mathrm{m}(n=7)$. Asterisks indicate $m d x$ greater than control myocytes $(P=0.008$ in untreated myocytes, $P=0.32$ in P188-treated myocytes and $P=0.044$ in nifedipine-treated myocytes). c, Maximum tolerated sarcomere length stretch in $m d x$ myocytes. Maximum tolerated sarcomere length is defined as the maximum length at which the myocyte is stable and fibrillations (Supplementary Video 3 ) are absent. Asterisk indicates $m d x$ less than all groups $(P<0.01)$; hash denotes $m d x+60 \mu \mathrm{M} P 188$ different from all groups $(P<0.05)$. Number of myocytes, $n=7$ (control), $7(m d x), 8(m d x+60 \mu \mathrm{M}$ P188), 7 ( $m d x+150 \mu$ M P188). d, FM 1-43 fluorescence after a single stretch in single cardiac myocytes. Summary of FM 1-43 fluorescence over time after a $20 \%$ stretch (arrow), showing mean change in voltage from baseline at the beginning of the stretch; number of myocytes, $n=7$ (control), 6 ( $m d x), 7$ $(m d x+150 \mu \mathrm{M}$ P188). For all graphs, data represent mean \pm s.e.m. 
myocyte length. The carbon fibres used in this study were rigid (40- $\mu \mathrm{m}$ diameter; $0.02 \mathrm{~m} \mathrm{~N}^{-1}$ compliance) and not altered by the active and passive tensions produced by single cardiac myocytes. Tension signal from the force transducer and length signal applied to the piezoelectric translator were recorded at 1,000$\mathrm{Hz}$ sampling rate with an analogue-to-digital recording system (Accura 100, Nicolet). Video recordings of the cardiac myocytes were digitized to measure cell extension and sarcomere length via images on a computer screen. For measurement of $\left[\mathrm{Ca}^{2+}\right]_{\mathrm{i}}$, myocytes were incubated with $5 \mu \mathrm{M}$ Fura-2-acetoxymethyl (AM) ester (Molecular Probes) and 0.02\% Pluronic F127 (Molecular Probes) for $4 \mathrm{~min}$ at $37^{\circ} \mathrm{C}$. Fura-2 excitation was sampled at $100 \mathrm{~Hz}$ using a microscope-based fluorescence spectrometer (Photon Technology International). $\left[\mathrm{Ca}^{2+}\right]_{\mathrm{i}}$ was determined using a previously established method ${ }^{23}$ after subtracting background fluorescence intensity. We investigated the effect of P188 $(150 \mu \mathrm{M} \text { in extracellular buffer unless otherwise stated })^{8}$ and nifedipine $(1 \mu \mathrm{M})$ on passive tension-extension characteristics and $[\mathrm{Ca}]_{\mathrm{i}}$ of
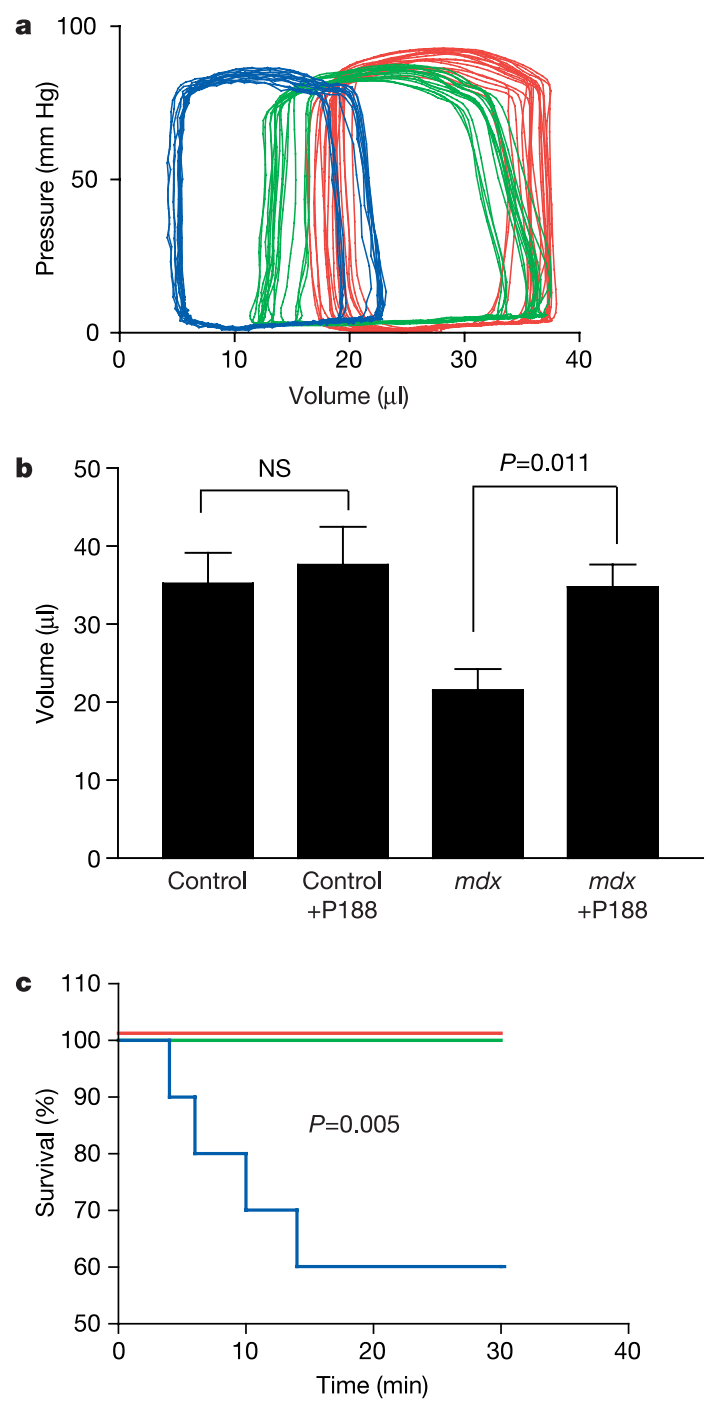

Figure 3 | Acute effects of P188 on in vivo haemodynamics and $m d x$ survival. a, Representative pressure-volume loops in control (red) and $m d x$ mice in the presence (green) or absence (blue) of acute infusion of P188.

b, Summary of left ventricular end-diastolic volumes immediately following infusion of P188 in control and $m d x$ mice. Values show mean \pm s.e.m., $n=13$ (control), 7 (control + P188), $13(m d x), 11(m d x+$ P188). NS, not significant. c, Kaplan-Meier survival analysis during infusion with $42 \mu \mathrm{g} \mathrm{kg}^{-1} \mathrm{~min}^{-1}$ dobutamine. Control (red), $m d x$ (blue), $m d x+\mathrm{P} 188$ (green). Mice were removed from the study when systolic pressures dropped below $60 \mathrm{~mm} \mathrm{Hg}$. Number of mice, $n=13$ (control), $10(m d x), 11$ $(m d x+$ P188). control and $m d x$ single cardiac myocytes. In another procedure, cardiac myocytes were stretched during the rising phase of twitch tension (lengthening contraction). Upon restoring sarcomere length, isometric twitch tension was again measured (post-stretch) and compared with pre-stretch twitch tension.

Membrane injury assay (FM 1-43 study). Single cardiac myocytes were stretched twice with $20 \%$ extension (1-s duration) of resting cell length (this extension is less than the critical level of stretch that results in contracture of $m d x$ cardiac myocytes) in HEPES-tyrode solution containing $1.8 \mathrm{mM} \mathrm{Ca}^{2+}$ and 2.5 $\mu \mathrm{M}$ FM 1-43 dye (Molecular Probes). In the initial stretch we confirmed the extension, and at the beginning of the second stretch $(t=0)$ we measured voltage output from a photomultiplier (PTi, Monmouth Junction) for $480 \mathrm{~s}$, representing fluorescence intensity of a $15 \times 50-\mu \mathrm{m}$ area on the myocytes.

Cardiac haemodynamics in vivo. Murine pressure-volume loops were acquired by previously described methods ${ }^{24}$. Briefly, anaesthetized mice were ventilated with $2 \%$ isoflourane and $98 \%$ oxygen. A thoracotomy and pericardiotomy were performed, and a pressure-volume catheter was inserted into the left ventricle via an apical stab. Before catheter insertion, mice received an intravenous infusion of $\sim 150 \mu \mathrm{l}$ of $10 \%$ human albumin with and without P188, at a rate of $\sim 200 \mu \mathrm{lgg}^{-1} \mathrm{~min}^{-1}$. After the collection of baseline haemodynamic data, dobutamine was infused at a dose of $42 \mu \mathrm{g} \mathrm{kg}^{-1} \mathrm{~min}^{-1}$ (ref. 25). The dose of P188 $\left(460 \mathrm{mg} \mathrm{kg}^{-1}\right)$ was shown to be effective at mitigating skeletal muscle damage following electrocution ${ }^{7}$. Acute cardiac failure was defined as a drop in left ventricular systolic pressure below $60 \mathrm{~mm} \mathrm{Hg}$, as below this level, rapid decompensation and death were frequent occurrences. Following the 30-min dobutamine challenge or the development of acute cardiac failure, volume measurements were calibrated as previously described $^{24}$.

Received 23 March; accepted 23 May 2005. Published online 17 July 2005.

1. Muntoni, F. Cardiomyopathy in muscular dystrophies. Curr. Opin. Neurol. 16, 577-583 (2003).

2. Emery, A. E. H. in Duchenne Muscular Dystrophy (ed. Emery, A. E. H.) 26-45 (Oxford Univ. Press, Oxford, 2003).

3. Finsterer, J. \& Stollberger, C. The heart in human dystrophinopathies. Cardiology 99, 1-19 (2003).

4. Rodriguez, E. K. et al. A method to reconstruct myocardial sarcomere lengths and orientations at transmural sites in beating canine hearts. Am. J. Physiol. 263, H293-H306 (1992).

5. Pasternak, C., Wong, S. \& Elson, E. L. Mechanical function of dystrophin in muscle cells. J. Cell Biol. 128, 355-361 (1995).

6. Wu, G. et al. Lipid corralling and poloxamer squeeze-out in membranes. Phys. Rev. Lett. 93, 028101 (2004).

7. Lee, R. C., River, L. P., Pan, F. S., Ji, L. \& Wollmann, R. L. Surfactant-induced sealing of electropermeabilized skeletal muscle membranes in vivo. Proc. Natl Acad. Sci. USA 89, 4524-4528 (1992).

8. Lee, R. C., Hannig, J., Matthews, K. L., Myerov, A. \& Chen, C. T. Pharmaceutical therapies for sealing of permeabilized cell membranes in electrical injuries. Ann. NY Acad. Sci. 888, 266-273 (1999).

9. Turner, P. R., Fong, P. Y., Denetclaw, W. F. \& Steinhardt, R. A. Increased calcium influx in dystrophic muscle. J. Cell Biol. 115, 1701-1712 (1991).

10. Togo, T., Alderton, J. M., Bi, G. Q. \& Steinhardt, R. A. The mechanism of facilitated cell membrane resealing. J. Cell Sci. 112, 719-731 (1999).

11. Bansal, D. et al. Defective membrane repair in dysferlin-deficient muscular dystrophy. Nature 423, 168-172 (2003).

12. Danialou, G. et al. Dystrophin-deficient cardiomyocytes are abnormally vulnerable to mechanical stress-induced contractile failure and injury. FASEB $J$. 15, 1655-1657 (2001).

13. Davis, D. B., Delmonte, A. J., Ly, C. T. \& McNally, E. M. Myoferlin, a candidate gene and potential modifier of muscular dystrophy. Hum. Mol. Genet. 9, 217-226 (2000).

14. Gregorevic, P. et al. Systemic delivery of genes to striated muscles using adeno-associated viral vectors. Nature Med. 10, 828-834 (2004).

15. Squire, S. et al. Prevention of pathology in $m d x$ mice by expression of utrophin: analysis using an inducible transgenic expression system. Hum. Mol. Genet. 11, 3333-3344 (2002).

16. Torrente, $\mathrm{Y}$. et al. Human circulating $\mathrm{AC} 133^{+}$stem cells restore dystrophin expression and ameliorate function in dystrophic skeletal muscle. J. Clin. Invest. 114, 182-195 (2004).

17. Goyenvalle, A. et al. Rescue of dystrophic muscle through U7 snRNA-mediated exon skipping. Science 306, 1796-1799 (2004).

18. Adams-Graves, P. et al. RheothRx (poloxamer 188) injection for the acute painful episode of sickle cell disease: a pilot study. Blood 90, 2041-2046 (1997).

19. Jewell, R. C., Khor, S. P., Kisor, D. F., LaCroix, K. A. \& Wargin, W. A. 
Pharmacokinetics of RheothRx injection in healthy male volunteers. J. Pharm. Sci. 86, 808-812 (1997)

20. Straub, V. \& Campbell, K. P. Muscular dystrophies and the dystrophinglycoprotein complex. Curr. Opin. Neurol. 10, 168-175 (1997).

21. Coutu, P., Bennett, C. N., Favre, E. G., Day, S. M. \& Metzger, J. M. Parvalbumin corrects slowed relaxation in adult cardiac myocytes expressing hypertrophic cardiomyopathy-linked $\alpha$-tropomyosin mutations. Circ. Res. 94, 1235-1241 (2004).

22. Yasuda, S. I. et al. A novel method to study contraction characteristics of a single cardiac myocyte using carbon fibers. Am. J. Physiol. Heart Circ. Physiol. 281, $\mathrm{H} 1442-\mathrm{H} 1446$ (2001).

23. Grynkiewicz, G., Poenie, M. \& Tsien, R. Y. A new generation of $\mathrm{Ca}^{2+}$ indicators with greatly improved fluorescence properties. J. Biol. Chem. 260, 3440-3450 (1985).

24. Michele, D. E., Gomez, C. A., Hong, K. E., Westfall, M. V. \& Metzger, J. M. Cardiac dysfunction in hypertrophic cardiomyopathy mutant tropomyosin mice is transgene-dependent, hypertrophy-independent, and improved by $\beta$ - blockade. Circ. Res. 91, 255-262 (2002).

25. Burger, A. J., Notarianni, M. P. \& Aronson, D. Safety and efficacy of an accelerated dobutamine stress echocardiography protocol in the evaluation of coronary artery disease. Am. J. Cardiol. 86, 825-829 (2000).

Supplementary Information is linked to the online version of the paper at www.nature.com/nature.

Acknowledgements This work was supported by a grant from the National Institute on Aging (J.M.M.). D.T. was supported by an NIH National Research Service Award, and S.M.D. was supported by an American Heart Association Fellow-to-Faculty Transition Award.

Author Information Reprints and permissions information is available at npg.nature.com/reprintsandpermissions. The authors declare no competing financial interests. Correspondence and requests for materials should be addressed to J.M. (metzgerj@umich.edu). 